\title{
Energy Efficient, Reconfigurable, Distributed Pulse Generation and Detection in UWB Impulse Radios
}

\author{
Jianyun Hu, Yunliang Zhu, Shang Wang, and Hui Wu \\ Laboratory for Advanced Integrated Circuits and Systems, Department of Electrical and Computer Engineering, \\ University of Rochester, Rochester, NY, contact: hwu@ece.rochester.edu
}

\begin{abstract}
Ultrafast pulse generation and detection are pivotal functions in ultra-wideband (UWB) impulse radios. This paper shows that digitally-assisted distributed circuit techniques provide an energy-efficient, reconfigurable solution for both functions, as demonstrated in two new circuits: a pulse generator that can generate reconfigurable pulse waveforms with subnanosecond time resolution, and a multi-GHz analog correlator that incorporates reconfigurable local template pulse generation. A UWB impulse radio was developed based on these new circuits with chip prototypes implemented in $0.18-\mu \mathrm{m}$ standard digital CMOS. It was characterized using UWB antennas, and achieved an energy efficiency of 25-pJ/pulse for the transmitter and 190-pJ/pulse for the receiver at $250 \mathrm{MHz}$ pulse rate in the measurement.
\end{abstract}

\section{INTRODUCTION}

Ultra-wideband (UWB) communication is emerging as the next-generation short-range wireless technology, particularly for wireless personal area networks [1]. Multi-band approaches, such as MB-OFDM [2], divide the large UWB bandwidth into several sub-bands, and operate within each sub-band as a conventional narrow-band radio. However, these systems require complex baseband circuitry and a multi-GHz tuning-range local oscillator [3], which significantly increase the hardware cost and power consumption. On the other hand, impulse radio UWB (IR-UWB) [4] utilizes a large portion of the full band from 3.1 to $10.6 \mathrm{GHz}$ without dividing it into sub-bands, and hence is highly scalable and enables truly software-defined radios. Since baseband signals are directly modulated on the ultrashort UWB pulses, IRUWB promises low transceiver complexity and low power consumption. Due to the large bandwidth, however, it remains challenging to design the analog front-end circuits that can efficiently generate, modulate, and detect the ultrashort IRUWB pulses using standard CMOS. In addition, it is highly desirable to use reconfigurable pulse waveforms in order to optimize the system performance on demand based channel environments, accommodate process, voltage and temperature (PVT) variations, and satisfy different regulatory requirements [5].

On the transmitter side, there are several approaches for IR-UWB pulse generation (Fig.1): carrier-based up-conversion [6], [7], direct pulse generation [8], [9], waveform synthesis based on high speed digital-to-analog converters (DAC) [10]. Carrier-based up-conversion defeats one of the major advantages of IR-UWB (no carrier) and results in large circuit complexity and power consumption. Direct pulse generation tends

Y. Zhu is now with Qualcomm Corp., San Diego, CA.

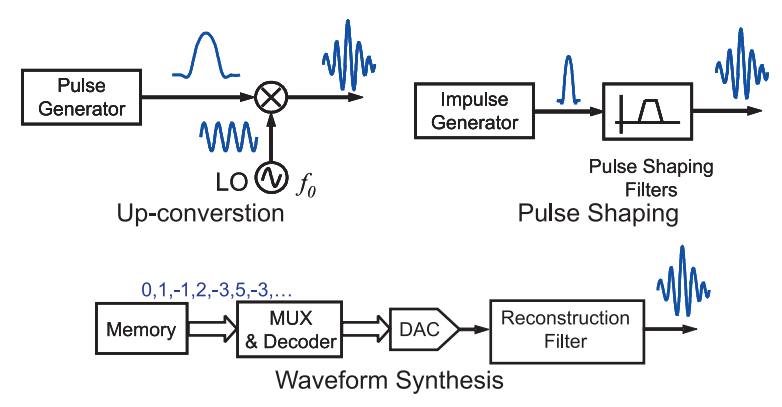

Fig. 1. Current IR-UWB pulse generation methods.

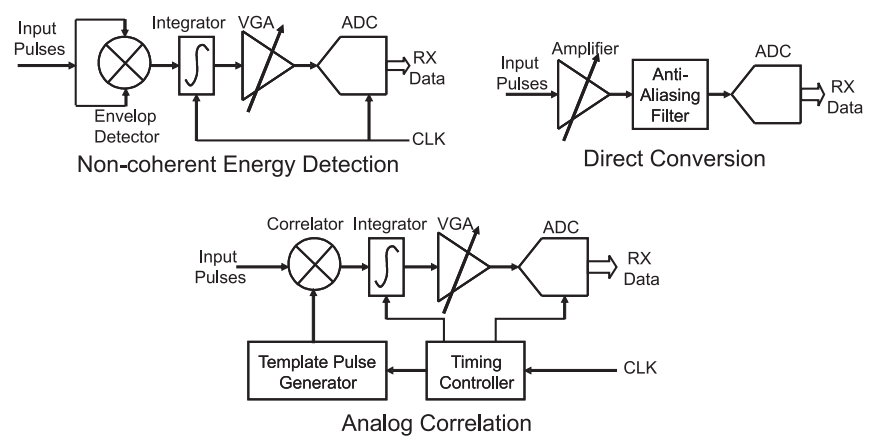

Fig. 2. Current IR-UWB pulse detection methods.

to have limited reconfigurability because the high-Q pulse shaping filter is typically implemented as an off-chip discrete passive filter. Waveform synthesis provides good time and amplitude resolution and is reconfigurable for different pulse shapes. However, the high sampling rate required presents a challenge to both the DAC implementation and the input data stream generation.

On the receiver side, direct conversion [11], analog correlation [8], [12], [13] and non-coherent energy detection [14][16] have been proposed for IR-UWB pulse detection (Fig.2). Since non-coherent receivers are more susceptible to noise and interference [15], coherent receivers can achieve higher data rate and hence are more desirable. The direct conversion receiver utilizes high speed analog-to-digital converters (ADC) to directly sample the UWB pulses. These high speed ADCs, however, consume a significant amount of power, and may substantially increase the system cost because they require advanced CMOS technologies. An analog correlation receiver moves the matched filter function from the digital domain to the analog front-end: the received pulses are correlated with a 
template pulse either locally generated or from a transmitted reference. This architecture reduces the required sampling frequency of the baseband ADC from Nyquist rate as in direct conversion to pulse rate, and hence significantly reduces the power consumption. The large bandwidth required by analog correlator and the accurate timing synchronization are two major challenges in this analog correlation architecture. Further, a reconfigurable template pulse generator is also needed to utilize different pulse waveforms.

To address these challenges in IR-UWB pulse generation and detection, this paper presents an energy efficient, reconfigurable UWB impulse radio based on digitally-assisted distributed circuit techniques.

\section{Distributed Pulse Generation And Detection}

Since IR-UWB uses low duty-cycle pulses with ultrashort (sub-nanosecond) time duration, the required sampling rate (e.g., 10 GSample/s for the low-band) is much higher than the pulse rate. Therefore, time interleaving, a circuit/architecture technique proven effective in Nyquist-rate data converters [17], is well suited for this purpose - with time interleaving, the sampling clock frequency $\left(f_{s}\right)$ and hence the circuit power consumption can be significantly lowered while maintaining a high sampling rate $\left(F_{s}=f_{s} * N\right.$, where $\mathrm{N}$ is the number of circuits time-interleaved). In order to satisfy the challenge of large bandwidth required in the time-interleaved circuits, distributed circuit techniques [18] can be applied. The distributed pulse generation (DWG) [19] and distributed pulse correlator (DPC) [20] shown below are time-interleaved, distributed circuits custom developed for UWB impulse radios.

The generic architecture of distributed UWB pulse generation and detection is shown in Fig.3. In the DWG, a UWB pulse is generated by combining impulses generated by multiple impulse generators in a time-interleaved fashion. The trigger signal is distributed to each impulse generator by the trigger distribution block, which enables impulses to be generated at specific sampling times. These impulses are then independently conditioned, i.e., changed to a desired pulse shape, polarity and amplitude, by a pulse conditioner block in each path, and then combined to form the output pulse waveform by a wideband pulse combining circuit. By changing the characteristics of each impulse generator and conditioner, different output pulse waveforms and spectra can be generated.

For the pulse detection, the DPC time-interleaves multiple pulsed multipliers in parallel. The input UWB pulse is distributed to these pulsed multipliers by the power distribution block. Each pulsed multiplier is triggered by an impulse generated by the impulse generator at a specific time, and multiplies the UWB pulse within the impulse duration by a software-defined tunable gain. This is equivalent to correlating the UWB pulse with a sequence of narrow impulses with tunable amplitude. The combination of these impulses essentially forms the built in reconfigurable local template pulse. All multiplier outputs are then summed and integrated to generate the overall correlation result. Since pulsed multipliers only

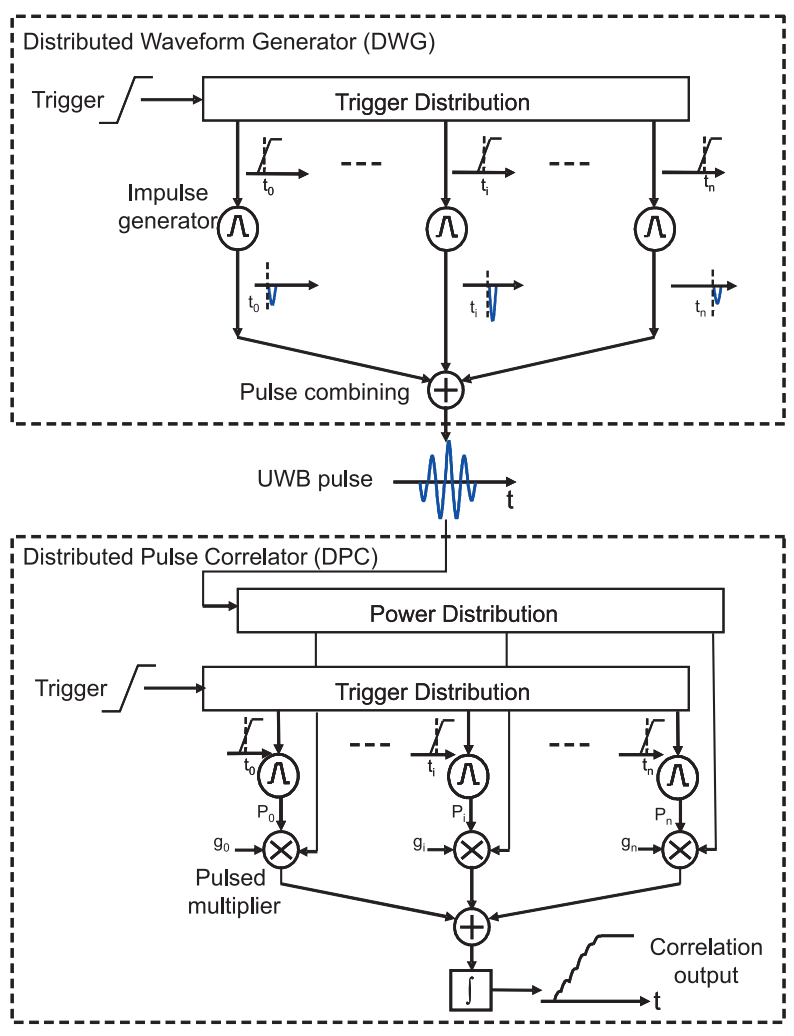

Fig. 3. Generic architecture of distributed UWB pulse generation and detection.

consume power when they are triggered, this architecture is especially energy efficient for low duty cycle IR-UWB pulse detection.

For the distributed pulse generation and detection, large analog bandwidth are only required for pulse combining in the DWG and power distribution in the DPC. To achieve the large analog bandwidth, the distributed circuit technique can be utilized without any active power consumption [21].

As is shown in Fig.3, both the DWG and DPC can also be treated as transversal finite impulse response (FIR) filters. The transversal structure of the DWG enables fully reconfigurability by changing the sampling time and pulse width of each impulse generator, and more importantly, by tuning the characteristics of each pulse conditioner. The transversal structure of the DPC enables reconfigurable local template pulses by changing the tunable gains, which makes the receiver more frequency and waveform agile. The transversal structrue of the DPC also enables the incorporation of linear equalization to address the issues of multipath and ISI.

The time-interleaved architecture required accurate timing control, which is mostly determined by the trigger distribution block in the DWG and DPC. Systematic delay mismatch and jitter in the trigger distribution can cause waveform distortion and detection error. A delay-locked loop (DLL)-based trigger distribution block is used to maintain an accurate timing control. 


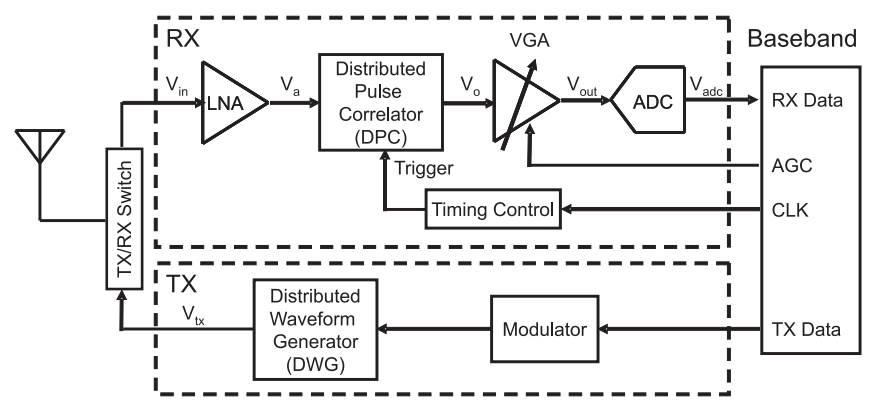

Fig. 4. IR-UWB transceiver architecture with DWG-based pulse generation and DPC-based pulse detection.

\section{PROTOTYPE IMPLEMENTATION}

Fig.4 shows the proposed transceiver architecture of the UWB impulse radio based on DWG and DPC. A modulator is added in the transmitter, which performs digital modulation on the low speed trigger signal to the DWG. In addition to the DPC, the analog correlation receiver consists of a lownoise amplifier (LNA) to amplify the received UWB signal, a timing control block to generate the trigger to the DPC and synchronize it with the incoming UWB pulse, a variable gain amplifier (VGA), and an ADC to amplify and digitize the DPC output.

A prototype UWB impulse radio was implemented in a 0.18- $\mu \mathrm{m}$ standard digital CMOS technology [19], [20]. In the transmitter, the DWG is constructed with an active delay line for trigger distribution, digital pulse generators for dualpolarity impulse generation, switched current sources for pulse amplitude control, and an on-chip transmission line for pulse combining. The modulator is constructed with a tunable delay line to implement pulse-position modulation (PPM) [19].

In the DPC-based receiver, the input UWB pulses are amplified by a resistive-feedback, single-to-differential UWB LNA first. The LNA has a 3-dB bandwidth from 2.3 to 9.8 $\mathrm{GHz}$, a maximum power gain of $10.1-\mathrm{dB}$, and a minimum noise figure of 3.9- $\mathrm{dB}$. The input and output return losses are above $6.5-\mathrm{dB}$ and $11-\mathrm{dB}$ over the 3-dB bandwidth, respectively. A two-stage cascaded VGA with a variable gain ranging from -10 to 32-dB further amplifies the DPC outputs to achieve a large dynamic range. A 3-bit full flash ADC converts the analog signal to the corresponding digital signal for baseband processing. The synchronization between Trigger and the incoming amplified signal $\mathrm{V}_{a}$ is performed by a DLLbased timing control block. A 6-bit controlled MUX is used to select one of the DLL outputs as the right timing for Trigger. During the synchronization phase, a training sequence is sent by the transmitter. The control signals of the MUX change incrementally to sweep the whole pulse period, and the proper timing can be set by detecting the acquisition according to the output of the ADC. In this prototype, the MUX is manually controlled, which can be carried out by the basedband processor in future implementation.

The chip photos of the transmitter and receiver chips are shown in Fig.5. The die size including pad frame is $2.8-\mathrm{mm}$
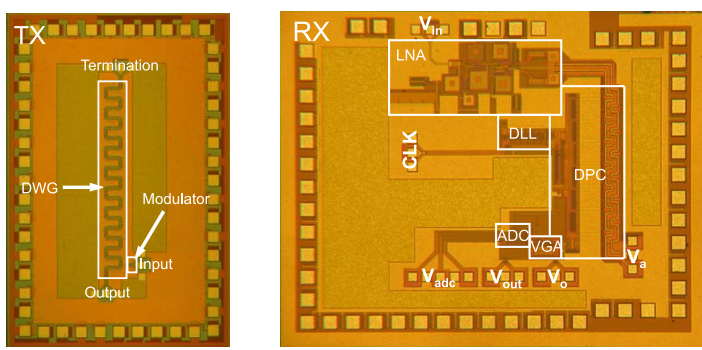

Fig. 5. Chip photos of the UWB impulse radio prototype.

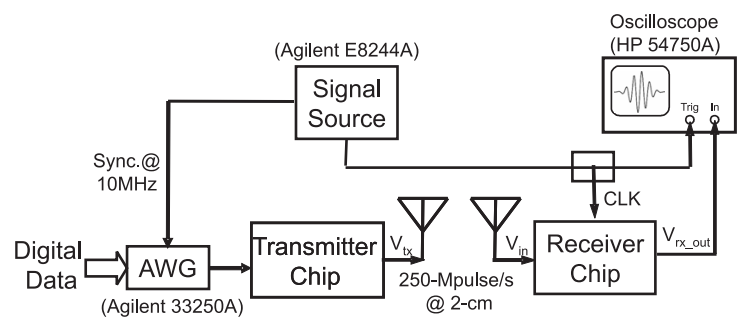

Fig. 6. Measurement setup for the UWB impulse radio test.

$\times 1.8-\mathrm{mm}$ and $3-\mathrm{mm} \times 2.4-\mathrm{mm}$ for transmitter and receiver, respectively. The active area is $0.32-\mathrm{mm}^{2}$ for transmitter and $1.23-\mathrm{mm}^{2}$ for receiver.

\section{Measurement Results}

The UWB impulse radio is characterized on wafer in time domain and frequency domain. In stand-alone transmitter test, the transmitter is characterized using the setup similar as Fig. 6, except that the transmitter output is directly connected to the oscilloscope and spectrum analyzer. Fig. 7 shows generated UWB waveforms of the transmitter (including the coefficient of each tap) with their frequency spectra. The waveform shown in first column is a monocycle with a duration of $0.5 \mathrm{~ns}$, generated by tap 3,4 . The corresponding spectrum has a $-10-\mathrm{dB}$ bandwidth of $6 \mathrm{GHz}$. The second column is a doublet with a duration of $0.5 \mathrm{~ns}$, generated by tap $1 \sim 3$. The corresponding spectrum has a center frequency of $3 \mathrm{GHz}$ and a $-10-\mathrm{dB}$ bandwidth of $5 \mathrm{GHz}$. Better frequency resolution can be achieved using more taps. As is shown in the third column, the complex UWB waveform (generated by 8 taps) has a duration of $0.8 \mathrm{~ns}$. It shows a well matched frequency spectrum with the FCC mask. The $-10-\mathrm{dB}$ bandwidth covers from $3 \mathrm{GHz}$ to $8 \mathrm{GHz}$, with the peak at about $6 \mathrm{GHz}$. These generated waveforms with different spectra demonstrate the reconfigurability of DWG. The reconfigurability of each impulse can also reduce the effect of the process, voltage and temperature (PVT) variations on the pulse shape. The transmitter is demonstrated to support a pulse rate for up to 2.5 $\mathrm{GHz}$, and the measured UWB waveform is well maintained for up to $1.5 \mathrm{GHz}$ pulse rate.

Fig. 8 shows the transmitter OOK modulation test when it is driven by a 32-bit, 32-Mb/s PRBS modulation signal, generated by an arbitrary waveform generator (AWG). The fluctuation on the waveform amplitude is caused by the limited 

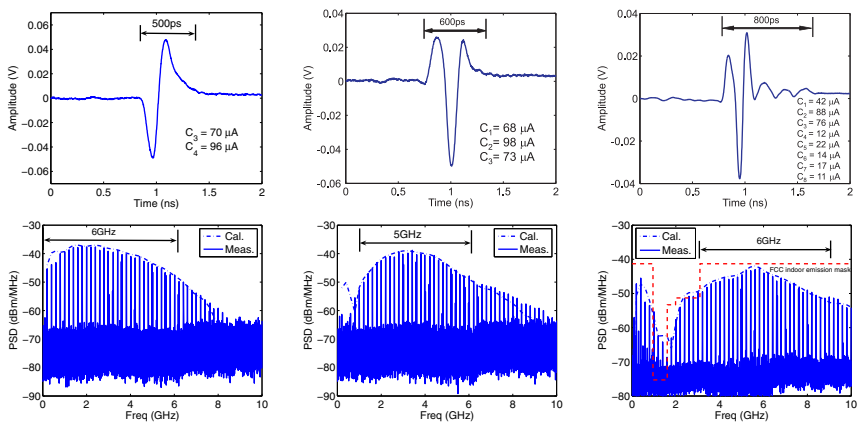

Fig. 7. Some measured waveforms and their spectra from the transmitter.
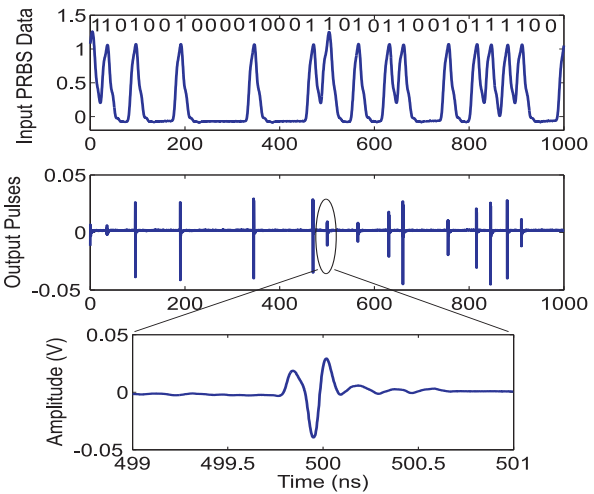

Fig. 8. Measurement results of the transmitter.

number of sampling points in the measurement window. The energy efficiency is $25-\mathrm{pJ} /$ pulse.

In the stand-alone receiver tests, a Gaussian-shape impulse with 70 ps pulse duration $\left(V_{i n}\right)$ is generated by an impulse generator and modulated by baseband data stream from the AWG. $V_{\text {in }}$ is converted into a differential UWB pulse with about $400 \mathrm{ps}$ pulse duration and $60 \mathrm{mV}$ voltage swing after passing the LNA.

To verify the functionality of DPC, each tap in the DPC is first used to correlate with different part of the UWB pulse independently by sweeping the trigger time delay. Correct output polarity and amplitude are observed. The conversion gain shows a good tuning linearity with the change of reference bias current. Then multiple taps are used to correlate with the input UWB pulses, the DPC output with correct timing and local template is shown in the first plot of Fig.9. The DPC output pulse show three phases during the operation: correlation, hold and reset. The hold phase is designed at about $1 \mathrm{~ns}$ for the following VGA and ADC, and the reset phase take about 2 ns. The relatively long reset time is caused by the parasitic capacitance of the integration capacitors, which can be further reduced in the improved design. Using the same local template, the DPC outputs (before the VGA) with different timing offset between the input UWB pulses and trigger signals are shown in the second plot of Fig.9. With no timing offset, the input UWB pulse is fully detected. With
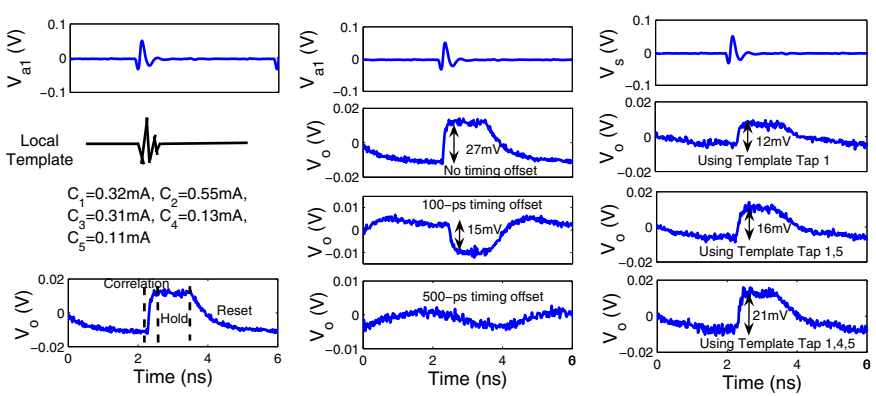

Fig. 9. Measured DPC outputs with correct timing and templates; different timing offset; different templates.

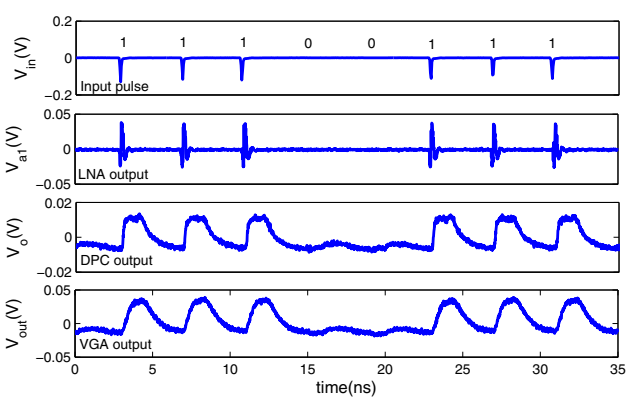

Fig. 10. Measurement results of the receiver with modulated data.

$100 \mathrm{ps}$ timing offset, since the polarity of each tap of template pulse is just opposite to the input pulse, a negative DPC output with smaller amplitude than the fully detected case is observed. With 500 ps timing offset, there is almost no correlation waveform observed. So accurate timing and synchronization are critical for the correct detection using DPC. The third plot of Fig.9 shows the correlation results for different local templates without timing offset: when tap 1 , tap 1,5 or tap $1,4,5$ are used, which only cover part of the UWB pulse, the correlation result has smaller amplitude compared to the fully detected case using tap 1 5 as shown in the second plot of Fig.9.

Fig.10 shows the complete receiver test results with maximum achievable pulse rate of $250 \mathrm{MHz}$ and with the modulated data stream from an AWG. The DPC achieves an energy efficiency of 40-pJ/pulse for a $250 \mathrm{MHz}$ pulse rate. Note that this figure of merit will remain constant even when the pulse rates is changed, which requires a different DLL frequency, since all its components are inherently duty-cycled. The LNA, VGA, ADC and DLL consume 12.2, 3.7, 2.9 and $2.4 \mathrm{~mA}$, respectively, from a $1.8 \mathrm{~V}$ power supply. Therefore, an energy efficiency of 190-pJ/pulse for $250 \mathrm{MHz}$ pulse rate is achieved.

The UWB impulse radio is demonstrated with two planar UWB monopole antennas [22], as shown in Fig. 6. Fig.11 shows the measurement results with different pulse rate and different transmitted waveforms. The first two in Fig.11 are measured with the same pulse rate of $250 \mathrm{MHz}$ while with different transmitted waveforms, and the third one is measured with a pulse rate of $150 \mathrm{MHz}$. At different pulse rate, by tuning the timing control block, synchronization between the received 

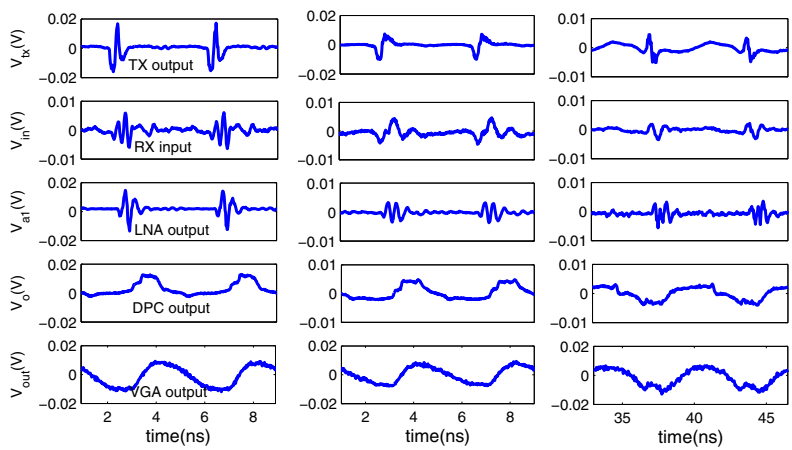

Fig. 11. Measurement results of the transceiver with different pulse rate and different transmitted waveforms.

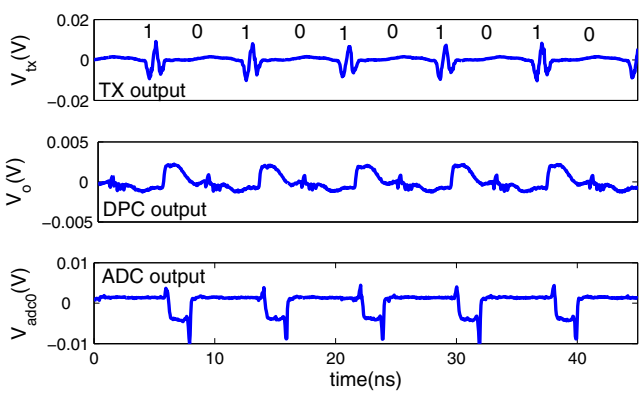

Fig. 12. Measurement results of the transceiver with modulated data.

pulses and local template timing can be achieved. Note that the UWB antennas and LNA change the pulse waveforms. By setting the polarity and coefficient of each DPC tap according to the received pulse waveforms, the correct pulse detection can be obtained. The UWB impulse radio is also demonstrated with the modulated data (Fig.12). Due to the limitation of the AWG, simple modulated data stream is utilized in this measurement. As is shown in Fig.12, the transmitted data can be successfully recovered at the receiver.

The transceiver performance is compared with other works in Table I. Among them, the DWG-based transmitter and DPC-based receiver achieve largest bandwidth, the highest achievable pulse rate and one of the best energy efficiency.

TABLE I

Performance Comparison With Other IR-UWB TRANSCEIVERS

\begin{tabular}{|c|c|c|c||c|c|c|c|}
\hline $\begin{array}{c}\text { Tx } \\
\text { Ref. }\end{array}$ & $\begin{array}{c}\text { BW } \\
(\mathrm{GHz})\end{array}$ & $\begin{array}{c}\text { Pulse } \\
\text { Rate } \\
(\mathrm{MHz})\end{array}$ & $\begin{array}{c}\text { Energy } \\
\text { Efficiency } \\
(\mathrm{pJ} / \text { pulse })\end{array}$ & $\begin{array}{c}\text { Rx } \\
\text { Ref. }\end{array}$ & $\begin{array}{c}\mathrm{BW} \\
(\mathrm{GHz})\end{array}$ & $\begin{array}{c}\text { Pulse } \\
\text { Rate } \\
(\mathrm{MHz})\end{array}$ & $\begin{array}{c}\text { Energy } \\
\text { Efficiency } \\
(\mathrm{pJ} / \mathrm{pulse})\end{array}$ \\
\hline$[6]$ & 2 & 500 & 210 & {$[8]$} & 2 & 200 & 405 \\
\hline$[8]$ & 2 & 400 & 190 & {$[12]$} & 3 & 50 & 2160 \\
\hline$[9]$ & 2 & 160 & 62.5 & {$[14]$} & 2 & 16.7 & 2500 \\
\hline$[14]$ & 2 & 16.7 & 43 & {$[15]$} & 2 & 100 & 144 \\
\hline $\begin{array}{c}\text { This } \\
\text { Work }\end{array}$ & 6 & 2500 & 25 & $\begin{array}{c}\text { This } \\
\text { Work }\end{array}$ & 7 & 250 & 190 \\
\hline
\end{tabular}

\section{CONCLUSION}

An energy efficient, reconfigurable UWB impulse-radio is demonstrated with chip prototypes implemented in a $0.18-\mu \mathrm{m}$ standard digital CMOS technology. Thanks to the distributed pulse generation and detection techniques, an energy efficiency of 25-pJ/pulse for transmitter and 190-pJ/pulse for receiver at $250 \mathrm{MHz}$ pulse rate in the measurement is achieved.

\section{ACKNOWLEDGMENT}

The authors would like to thank B. Chatterjee, A. Bahai, P. Holloway, M. Bohsali, J. Yu, A. Shah, V. Abellera, P. Misich, and J. Wan of National Semiconductor for their support in chip fabrication. The authors also would like to thank Fujitsu Component America, Inc. for providing UWB antennas. This work is in part supported by the National Science Foundation (NSF) under Contract 0829915.

\section{REFERENCES}

[1] IEEE std 802.15.4a. 2007.

[2] A. Batra et al. Multiband OFDM physical layer specification. WiMedia Alliance, Release 1.1, July 2005.

[3] H. Zheng et al. A $3.1 \mathrm{GHz}-8.0 \mathrm{GHz}$ Single-Chip Transceiver for MBOFDM UWB in 0.18-um CMOS Process. IEEE J. Solid-State Circuits, 44(2):414-426, Feb. 2009.

[4] M.Z. Win and R.A. Scholtz. Impulse radio: How it works. IEEE Communication Letters, 2:36-38, 1998.

[5] Y. Zhu, J.D. Zuegel, J.R. Marciante and H. Wu. Distributed Waveform Generator: A New Circuit Technique for Ultra-Wideband Pulse Generation, Shaping and Modulation. IEEE J. Solid-State Circuits, 44(3):808823, Mar. 2009.

[6] S. Lida et al. A 3.1 to $5 \mathrm{GHz}$ CMOS DSSS UWB transceiver for WPANs. In IEEE Int. Solid-State Circuits Conf. Dig. Tech. Papers, pages 214-215, 2005.

[7] D.D. Wentzloff and A.P. Chandrakasan. Gaussian Pulse Generators for Subbanded Ultra-wideband Transmitter. In IEEE Trans. Microwave Theory Tech., 54(4):1647-1655, Apr. 2006.

[8] Y. Zheng et al. A CMOS Carrier-less UWB Transceiver for WPAN Applications. In IEEE Int. Solid-State Circuits Conf. Dig. Tech. Papers, pages 116-117, 2006.

[9] L. Smaini et al. Single-chip cmos pulse generator for uwb systems. IEEE J. Solid-State Circuits, 41(7):1551-1561, Jul 2006.

[10] D. Baranauskas and D. Zelenin. A $0.36 \mathrm{~W} 6 \mathrm{~b}$ up to $20 \mathrm{GS} / \mathrm{s}$ DAC for UWB Wave Formation. In IEEE Int. Solid-State Circuits Conf. Dig. Tech. Papers, pages 580-581, 2006.

[11] I. O'Donnel et al. An Integrated, Low-Power, Ultra-Wideband Transceiver Architecture for Low-Rate, Indoor Wireless Systems. In IEEE CAS Workshop on Wireless Comm. and Networking, Sep. 2002.

[12] J. He and Y.P. Zhang. A CMOS Ultra-Wideband Impulse Radio Transceiver for Interchip Wireless Communications. In IEEE ICUWB., pages $626-631,2007$.

[13] R. Hoctor and H. Tomlinson. Delay-hopped transmitted-reference RF communications. In IEEE ICUWB., pages 265-269, 2002.

[14] D.D. Wentzloff et al. Energy Efficient Pulsed-UWB CMOS Circuits and Systems. In IEEE ICUWB., pages 282-287, 2007.

[15] T.-A. Phan et al. Low-Power CMOS Energy Detection Transceiver for UWB Impulse Radio System. In IEEE Custom Integrated Circuits Conf. Dig. Tech. Papers, pages 675-678, 2007.

[16] L. Stoica et al. Impulse Radio based Non-Coherent UWB Transceiver Architectures - An Example. In IEEE ICUWB., pages 483-488, 2006.

[17] W.C. Black and D.A. Hodges. Time interleaved converter arrays. IEEE J. Solid-State Circuits, SC-15(12):1022-1029, Dec. 1980.

[18] T. Wong. Fundamentals of Distributed Amplification. Artech House, 1993.

[19] Y. Zhu, J. Hu, and H. Wu. A 2.5 Gpulse/s, 25 pJ/pulse, $0.18 \mu \mathrm{m}$ CMOS Impulse Radio UWB Transmitter Based on Dual-Polarity Distributed Waveform Generator. In IEEE Topical Meeting on Silicon Monolithic Integrated Circuits in RF Systems, pages 225-228, 2009.

[20] J. Hu, Y. Zhu, S. Wang, and H. Wu. A 0.17-nJ/Pulse IR-UWB Receiver Based on Distributed Pulse Correlator in 0.18-um Digital CMOS. In IEEE Radio Freq. Integrated Circuit Symp., pages 543-546, 2009.

[21] Y. Zhu, S. Wang, and H. Wu. Multilayer Coplanar Waveguide Transmission Lines Compatible with Standard Digital Silicon Technologies. In IEEE Int. Microwave Symposium, pages 1567-1570, 2007.

[22] http://www.fujitsu.com/us/services/edevices/components/input/uwb.html. 